\title{
Identifying Malicious Accounts in Social Media Based on Online Promotions
}

\author{
Gudapati Navya, Duvvada Rajaseswara Rao
}

\begin{abstract}
There is a developing number of individuals who hold accounts via web-based networking media stages however conceal their character for pernicious purposes. Tragically, almost no research has been done to date to distinguish counterfeit characters made by people, particularly so via web-based networking media stages. Online social media step by step incorporate monetary capacities by permitting the utilization of genuine and virtual money, filling in as new stages to have an assortment of organizations, for example, online limited time occasions, where clients can become virtual assesses as a compensation for going to such occasions. Both NSOs and business stakeholders are essentially concerned when Attackers actualize an assortment of records to gather virtual money from these occasions, making these occasions insufficient and the outcome in critical budgetary misfortune. It turns out to be critical to proactively identify these malignant records before on the web and special exercises in this manner they decline their need to be remunerated. In this paper, we have present a new framework, called ProGuard, for accomplish this by deliberately coordinating highlights that describe accounts from three viewpoints, including their overall conduct, their top-up designs and the utilization of their cash. We directed various experiments dependent on information gathered by Ten cent QQ, a world chief OSN with coordinated budgetary administration exercises. Exploratory outcomes have indicated that our framework is equipped for accomplishing a high recognition pace of $96.67 \%$ at a low false positive pace of $0.3 \%$.
\end{abstract}

Keywords: Malicious Accounts, ProGuard, Recognition, Social Media, Virtual Money, Ten cent $Q Q$

\section{INTRODUCTION}

The significant development of social media networks has allowed to countless people to share information on a scale scarcely believable during the previous decade. Existing examinations that social media networks presently pronouncedly affect society and human day by day life. For instance, in colleges, social media networks can be utilized to impart, spread grounds news, make declarations and give understudies helpful information. Social media additionally has a significant job in legislative issues, spreading law based thoughts across worldwide outskirts. In addition, social media

Revised Manuscript Received on July 30, 2020

* Correspondence Author

Gudapati Navya*, CSE, V R Siddhartha Engineering College, Vijayawada, India. E-mail: navyagudapati999@gmail.com

Dr. Duvvada Rajeswara Rao, CSE, V R Siddhartha Engineering College, Vijayawada, India. E-mail: rajeshpitam@gmail.com

(C) The Authors. Published by Blue Eyes Intelligence Engineering and Sciences Publication (BEIESP). This is an open access article under the CC BY-NC-ND license (http://creativecommons.org/licenses/by-nc-nd/4.0/) is significant for promoting; since it is a ground-breaking route for organizations to arrive at clients and to drive crowd commitment. Recognizing traded off personal accounts should be possible by catching the activity history of a client and attempting to identify designs in his/her conduct. At that point, bargained accounts will be the ones that go amiss altogether from the identified examples. Be that as it may, personal social accounts show change in conduct after some time; contingent upon the client's personality, state of mind and leisure time. In this manner, so as to accomplish the recently expressed target, we identify stable personal conduct standards after some time in certain utilization highlights, for example, language, activity time, activity type, and so forth.

As personal accounts are conflicting, every client will have an alternate arrangement of highlights that can extraordinarily indicate their use design. In this manner, we characterize a load for every client's highlights and a scoring component that is utilized to make a social profile for every client. We likewise direct a study to gather client's socioeconomics and other personal data, to have the option to know every clients personality, instructive status, work status, interests, and activity rates. The conduct profiles that are created can be utilized to identify hacking endeavors, when the activity goes amiss from a client's example by in excess of a particular edge. Online social systems that incorporate virtual currency go about as an appealing stage for different organizations, where intelligent online advancement is one of the most dynamic. Specifically, a client, regularly spoke to by their OSN account, can win a prize as virtual currency by taking part in online limited time activities sorted out by corporate substances. This virtual currency permits an online advancement model that takes into account a wide range, offers direct monetary impetuses to end clients and, meanwhile, limits the cooperation's between business elements and money related circumstances. Such unsafe activities will significantly bargain the adequacy of limited time activities, quickly dropping the viability of special ventures by business elements and, meanwhile, harming the notoriety of the NSOs. Consequently, it is indispensably imperative to distinguish accounts constrained by aggressors in online limited time activities. To start with, assailants don't need to produce malignant substance to dispatch fruitful assaults. Furthermore, effective assaults must not be founded on social structures. To be increasingly explicit, keeping up dynamic social structures doesn't profit assailants, which is on a very basic level not quite the same as well-known assaults, for example, spammers on online social systems.

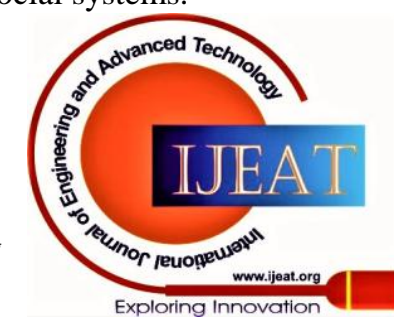




\section{Identifying Malicious Accounts in Social Media Based on Online Promotions}

These two difficulties make the identification of these malignant OSN accounts on a very basic level not quite the same as the location of customary assaults like spam and phishing. These highlights expect to describe a record from three perspectives, including I) its overall utilization profile, ii) how a record gathers virtual currency, iii) how virtual currency is spent. We assessed our framework utilizing information gathered by Tencent QQ, a main online social system in China that utilizes a generally acknowledged virtual currency, to help online money related activities for a huge assemblage of 899 million accounts. Dynamic.

\section{RELATED WORK}

In this section, we have discussed on different existing studies regarding on Identifying malicious accounts in social media. Mosab Khayat et.al, [1] presented a paper called as VASSL: A Visual Analytics Toolkit for Social Spambot Labeling. In this paper, they present VASSL, a visual examination framework that aids the way toward identifying and naming spambots. Our device improves the presentation and adaptability of manual marking by giving different associated sees and using dimensionality decrease, opinion investigation and point demonstrating, empowering bits of knowledge for the distinguishing proof of spambots. The framework permits clients to choose and investigate gatherings of records in an intelligent way, which empowers the identification of spambots that may not be recognized when analyzed exclusively. JAN ELOFF et.al, [2] proposed a method titled as Using Machine Learning to Detect Fake Identities: Bots vs Humans. Interestingly, numerous models exist of situations where records made by bots phony or PCs have been identified effectively utilizing AI models. On account of bots these AI models were reliant on utilizing designed highlights, for example, the 'companion to-adherents proportion'. These highlights were built from traits, for example, 'companion check' and 'devotee tally', which are straightforwardly accessible in the record profiles on SMPs. The exploration talked about in this paper applies these equivalent built highlights to a lot of phony human records in the desire for propelling the effective recognition of phony characters made by people via web-based networking media stages. Manuel Egele et.al, [3] introduced a paper named as Towards Detecting Compromised Accounts on Social Networks. In this work, they show how they can utilize comparative strategies to recognize bargains of individual high-profile accounts. High-profile accounts much of the time have one trademark that makes this location dependable - they show reliable conduct after some time. They show that our framework, were it conveyed, would have had the option to distinguish and forestall three genuine assaults against famous organizations and news offices. Besides, our framework, rather than well-known media, would not have fallen for an organized trade off impelled by a US eatery network for exposure reasons. Rana Mohamed Eisa et.al, [4] introduced a paper called as SOS: Save Our Social Network Accounts. In this paper, we propose a novel procedure dependent on emotional rationale and AI strategies to recognize and separate the most exceptionally characterizing features of each close to home record. These features are then used to fabricate a social profile for each client, which can be utilized to recognize peculiarities. We directed 2 examinations, gathered information from 47 Facebook clients and 616 Twitter clients. They extricated features from the gathered information and allotted each element a load as a size of significance as indicated by the client's deviation in this element. Sarah Khaled et.al, [5] proposed a paper called as Detecting Fake Accounts on Social Media. In this paper, another algorithm, SVM-NN, is proposed to give proficient location to fake Twitter accounts and bots, highlight choice and measurement decrease strategies were applied. AI order algorithms were utilized to choose the objective accounts character genuine or fake, those algorithms were bolster vector machine and our recently evolved algorithm, SVM-NN. The proposed algorithm utilizes less number of highlights, while as yet having the option to effectively group about $98 \%$ of the accounts of our preparation dataset. Mehmet Kaya et.al, [6] presented a paper named as Visualization of the social bot's fingerprints. In this paper, they research approaches to choose the best highlights from a dataset for approved order of various kinds of online networking accounts. Naman Singh et.al, [7] presented a paper named as Detection of Fake Profile in Online Social Networks Using Machine Learning. There are various situations where delivered accounts have been viably recognized using machine adjusting methods anyway the measure of examination work is exceptionally low to perceive fake characters made by individuals. For bots the ML models utilized different highlights to figure the no. of devotees to the no. of companions that a record has via web-based networking media stages. Sicong Shao et.al, [8] proposed a paper called as Automated Twitter Author Clustering with Unsupervised Learning for Social Media Forensics. In this paper, we propose a novel methodology through a blend of highlight extraction techniques and afterward convert high dimensional information to bit lattice for Twitter creator grouping. The test results show that our methodology can be utilized to adequately distinguish the gatherings among more than one hundred Twitter pseudonyms even without knowing the quantity of creators. Antu Mary Kuruvilla et.al, [9] presented a paper called as A Detection System to Counter Identity Deception in Social Media Applications. Internet based life administrations, for example, communitarian task's single client continually makes numerous accounts with various record names not long after a square has been applied. The blocked individual who makes different accounts is called sock manikin. Current system for recognizing misleading depend on human double dealing discovery (e.g., discourse or text).Although these strategy have high identification precision, yet it can't be applied in databases with enormous volumes of information. So they are computationally wasteful. Yadong Zhou et.al, [10] introduced a paper called as ProGuard: Detecting Malicious Accounts in Social-Network-Based Online Promotions. In this paper, we propose a novel system, namely ProGuard, to accomplish this objective by systematically integrating features that characterize accounts from three perspectives including their general behaviors, their recharging patterns, and the usage of their currency.

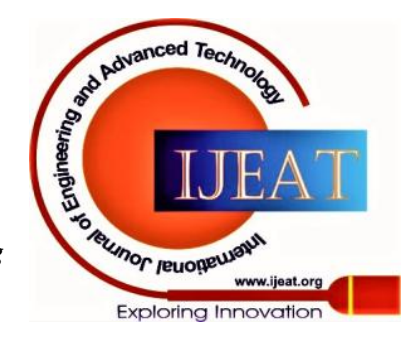




\section{METHODOLOGY}

In this section, we have describe the structure and working of our proposed methodology.

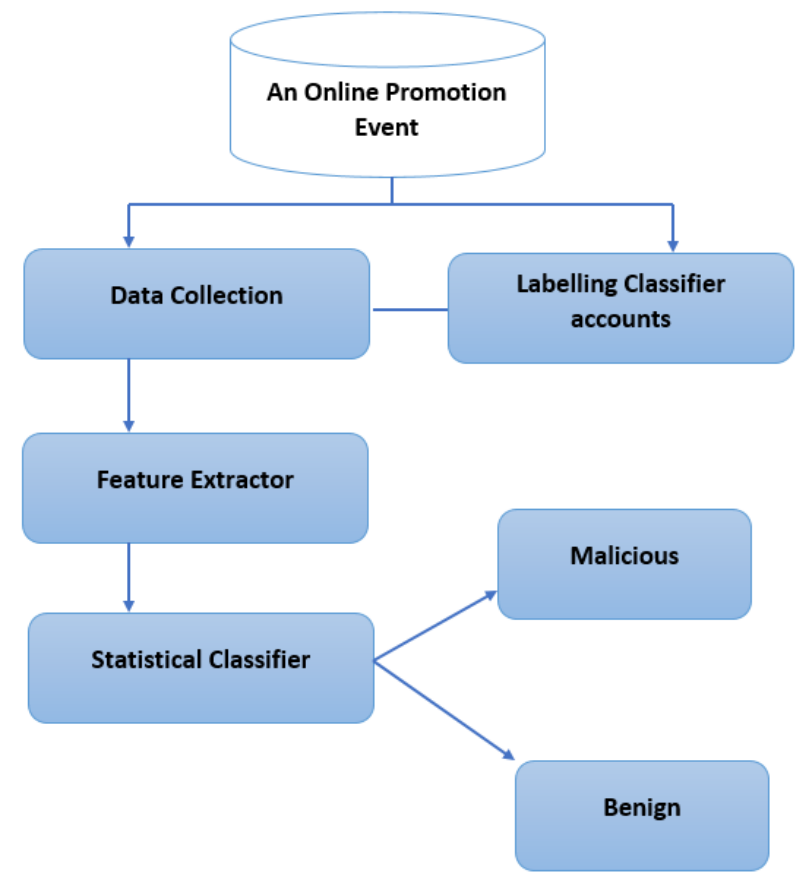

Fig 1. The structure of our proposed system

The proposed system consists of the following phases they are,

- Data set Collection

- ProGuard

- General Behaviors

- Currency Collection

- Currency Usage

\section{A. Data Set Collection}

In this, we have gathered marked information from Tencent QQ, a main Chinese online interpersonal organization that offers an assortment of administrations, for example, text, voice talk, and web based games, web based shopping, and web based business. The dataset that are utilizing here thoroughly comprises of 56,000 records where whole dataset is isolated into 28,000 noxious records and 28,000 benevolent records. This kind of dataset fill in as an even dataset for preparing a factual classifier

\section{B. ProGuard}

ProGuard is made out of two stages, in particular the preparation stage and the recognition stage. In the preparation stage, a measurable classifier is found out from a lot of pre-marked noxious and amiable records. In the location stage, an obscure record will initially be changed over to an element vector and afterward examined by the measurable classifier to survey its vindictiveness.

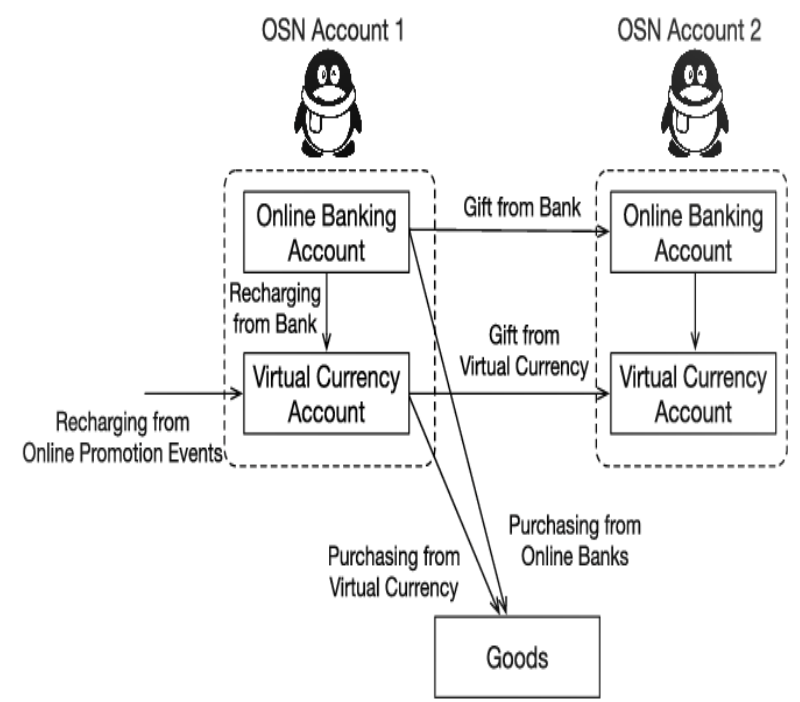

Fig 2. Overview of our proposed system

\section{General Behaviors}

Benign records are generally utilized by standard clients for assortment of exercises, for example, talking, photograph sharing, and financial exercises. Interestingly, malignant records are bound to be driven by online advancement occasions. Along these lines, the kind records will in general be all the more socially dynamic contrasted with malevolent records.

The highlights proposed as a rule practices are

- The Ratio of Active Days

- The Number of Friends

- The Number of Services Purchased by an Account

\section{Collection of Currency}

The malicious records under scrutiny center on utilizing on the web advancement exercises to gather virtual cash. Interestingly, favorable clients are probably going to get virtual cash from numerous assets. We propose two highlights to describe this pattern from two viewpoints including the measure of energizing and the significant hotspots for reviving.

- The Average Recharge Amount of Virtual Currency

- The Percentage of Recharge from Promotion Activities

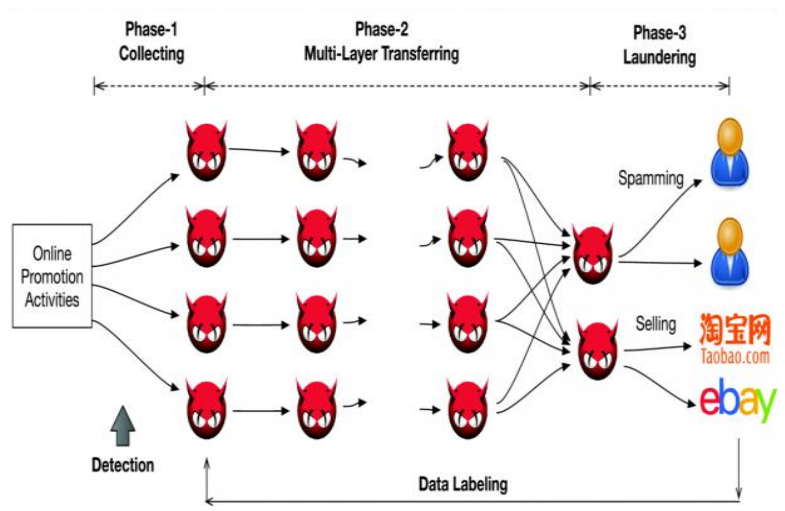

Fig 3. Virtual Currency Flow for Malicious OSN Accounts

Published By:

Blue Eyes Intelligence Engineering \& Sciences Publication

(C) Cobvriaht: All riahts reserved.

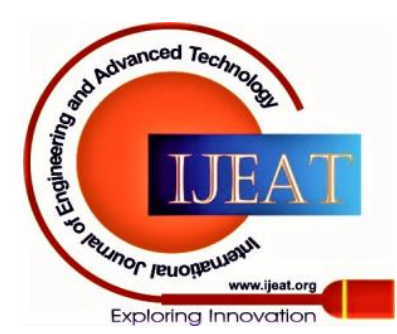




\section{Identifying Malicious Accounts in Social Media Based on Online Promotions}

\section{E. Collection of Currency}

Aggressors' definitive target is to adapt the virtual cash. Conversely, favourable clients utilize their virtual money in significantly more diversified ways.

Here, we propose three highlights:

- Total Amount of Expenditure

- The Percentage of Expenditure from Banks

- The Percentage of Expenditure as Rewards.

\section{RESULTS AND DISCUSSION}

We performed broad assessment of ProGuard, which centers on the general location exactness, the significance of each element, and the connection among these highlights. For this assessment, we utilized absolutely 56,000 records whose whole dataset is separated into 28,000 noxious records and 28,000 generous records. Such information fill in as an even dataset for preparing a measurable classifier. The calculation utilized here is positioning capacities from outstanding task at hand. This calculation that starts a streamlining agent to process guests advanced character boundaries on client's assets and give a course of events examination of occasions for better dynamic of pernicious user identification

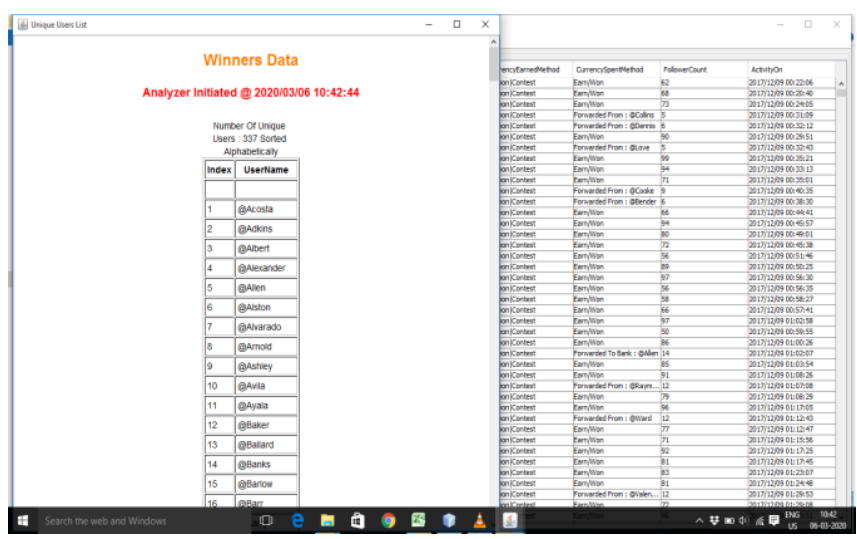

Fig 4. The Output of winners' data where the number of unique users are 337 users sorted alphabetically.

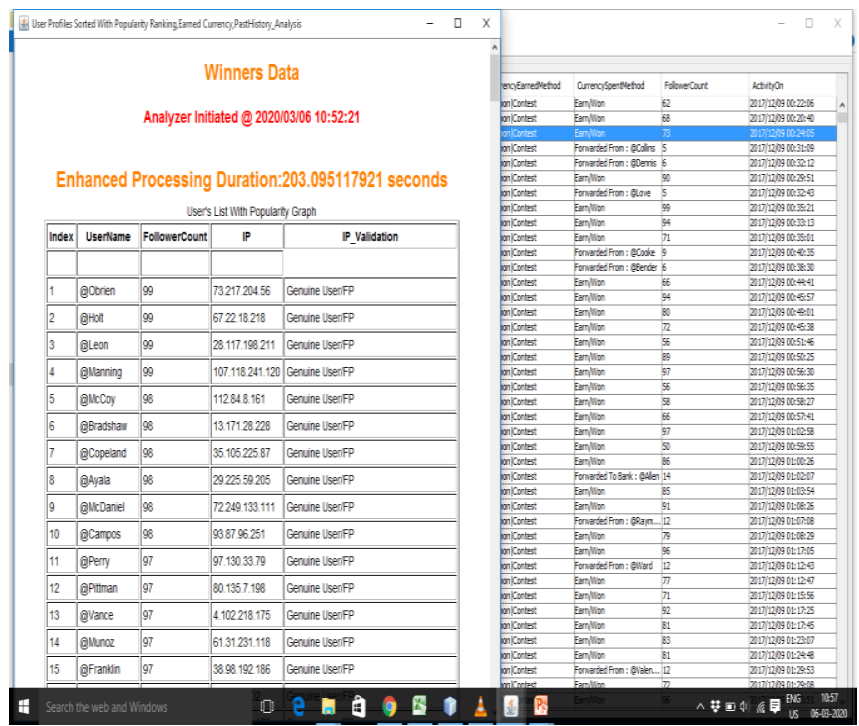

Fig 5. The Output of winners' data in a contest conducted to earn or won the contest and to check whether the user is genuine or not.

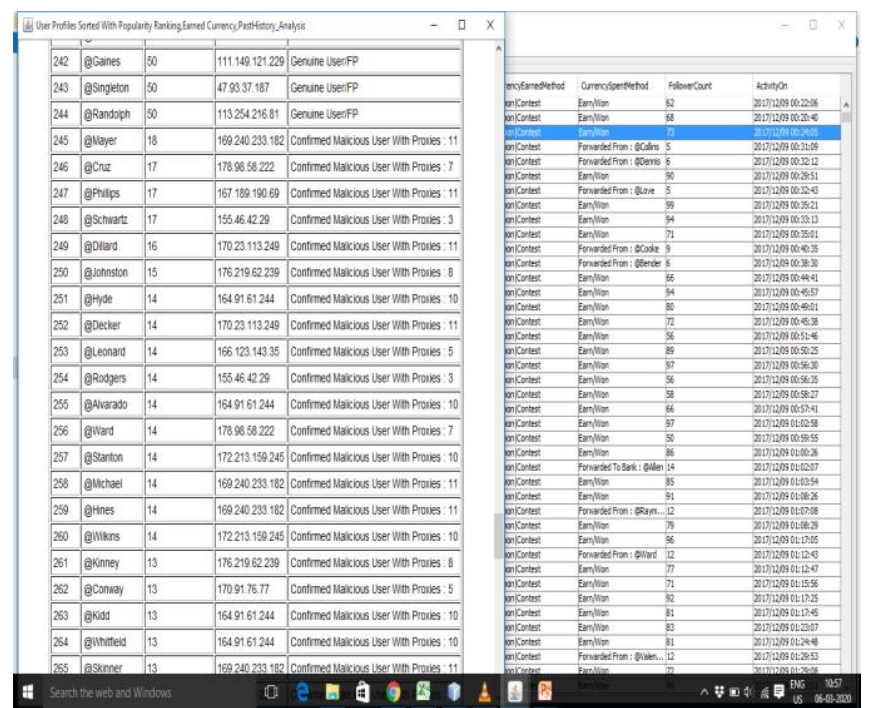

Fig 6. The Output of winners' data conducted in a context to check how many winners have confirmed malicious user with proxies

\section{CONCLUSION}

In this paper, we have performed broad assessment of ProGuard, which centers on the general location exactness, the significance of each element, and the connection among these highlights. For this assessment, we utilized absolutely 56,000 records whose whole dataset is separated into 28,000 noxious records and 28,000 generous records. Also, this paper presents another framework, ProGuard, to naturally distinguish pernicious web based life accounts taking part in online advancement exercises. ProGuard exploits three classes of highlights including general conduct, virtual cash assortment and utilization of virtual money. Exploratory outcomes dependent on the stamped information gathered by Tencent QQ, a world-driving online life organize organization, showed the exactness of identification of ProGuard, which accomplished a high discovery pace of 95\% given a bogus positive rate very low $0.1 \%$.

\section{REFERENCES}

1. Khayat M, Karimzadeh M, Zhao J, Ebert DS. VASSL: A Visual Analytics Toolkit for Social Spambot Labeling. IEEE transactions on visualization and computer graphics. 2019 Aug 19;26(1):874-83.

2. Van Der Walt E, Eloff J. Using machine learning to detect fake identities: Bots vs humans. IEEE Access. 2018 Jan 22;6:6540-9.

3. Egele M, Stringhini G, Kruegel C, Vigna G. Towards detecting compromised accounts on social networks. IEEE Transactions on Dependable and Secure Computing. 2015 Sep 17;14(4):447-60.

4. Eisa RM, Labib M, ElMougy A. SOS: Save Our Social Network Accounts. In2019 IEEE 17th World Symposium on Applied Machine Intelligence and Informatics (SAMI) 2019 Jan 24 (pp. 43-48). IEEE.

5. Khaled S, El-Tazi N, Mokhtar HM. Detecting Fake Accounts on Social Media. In2018 IEEE International Conference on Big Data (Big Data) 2018 Dec 10 (pp. 3672-3681). IEEE.

6. Kaya M, Conley S, Varol A. Visualization of the social bot's fingerprints. In2016 4th International Symposium on Digital Forensic and Security (ISDFS) 2016 Apr 25 (pp. 161-166). IEEE.

7. Singh N, Sharma T, Thakral A, Choudhury T. Detection of fake profile in online social networks using machine learning. In2018 International Conference on Advances in Computing and Communication Engineering (ICACCE) 2018 Jun 22 (pp. 231-234). IEEE.

Published By:

Blue Eyes Intelligence Engineering

\& Sciences Publication

(C) Convriaht: All riahts reserved.

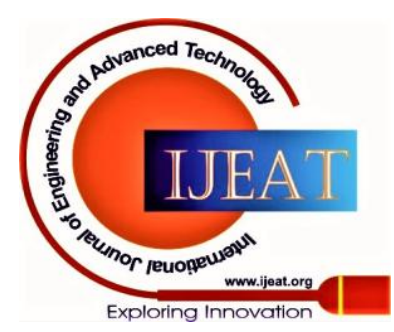


8. Shao S, Tunc C, Al-Shawi A, Hariri S. Automated Twitter Author Clustering with Unsupervised Learning for Social Media Forensics. In2019 IEEE/ACS 16th International Conference on Computer Systems and Applications (AICCSA) 2019 Nov 3 (pp. 1-8). IEEE.

9. Kuruvilla AM, Varghese S. A detection system to counter identity deception in social media applications. In2015 International Conference on Circuits, Power and Computing Technologies [ICCPCT-2015] 2015 Mar 19 (pp. 1-5). IEEE.

10. Zhou Y, Kim DW, Zhang J, Liu L, Jin H, Jin H, Liu T. Proguard: Detecting malicious accounts in social-network-based online promotions. IEEE Access. 2017 Jan 17;5:1990-9.

\section{AUTHORS PROFILE}

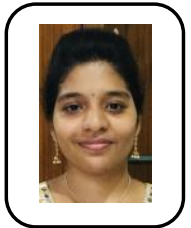

Gudapati Navya, studying Master of Technology, Department of Computer Science and Engineering, Velagapudi Ramakrishna Siddhartha Engineering College, Vijayawada. Obtained B.Tech Degree in Computer Science and Engineering in Velagapudi Ramakrishna Siddhartha Engineering College, Vijayawada, and Andhra Pradesh, India in 2016.

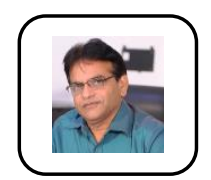

Duvvada Rajeswara Rao, Head of Department of Computer Science and Engineering (CSE), HOD, Velagapudi Ramakrishna Siddhartha Engineering College, Vijayawada. He is qualified in Ph.D. in Computer Science and Engineering. He has 25 years of teaching experience and He published more than 62 journal papers and 5 international conference papers.

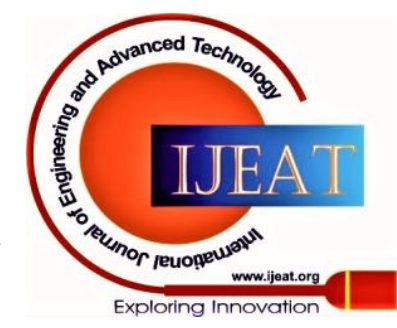

